\title{
Reflection! What reflection?
}

\author{
Unwanted reflections can severely limit the performance of optical components. David Gevaux \\ spoke to Fred Schubert from Rensselaer Polytechnic Institute about how his nanomaterials with a \\ refractive index almost equal to that of air can help.
}

Why are you conducting this research? In the past, I have often asked myself: what is the most important parameter in optics and in optical materials? I have come to the conclusion that it is the refractive index. There is a great need for low-refractive-index materials, but when it comes to viable thin films, the choice is quite limited. We have glass at 1.45 or 1.46 , but in the range of 1 to 1.4 there is not much available. Aerogels - highly porous materials that are spun onto surfaces as a film - are one option, but it's hard to control their thickness and grow very thin films. The problem is that spin-on technology is most suited for a film thickness larger than about half a micrometre, but for many applications the film thickness needs to be a quarter of the wavelength, around $100 \mathrm{~nm}$ for visible light.

Tell me about your low-index materials. In our method, we deposit silica nanorods by evaporation. We get very good control over the refractive index by varying the angle of incidence. We know that at, let's say, an $80^{\circ}$ deposition angle, we get a refractive index of about 1.1 , at $85^{\circ}$ we get 1.07 and at $87^{\circ}$ we get 1.05 , which I think is a world-record low. So we have a clear relationship between our input parameters and the resulting refractive index. Therefore, it is my opinion that our research into obliquely deposited nanorods represents an enormous milestone because we are filling a gap where no viable thin-film material existed before. We are creating a new class of optical materials, and the graded-index antireflection coatings that we describe in this issue are just one example of the potential applications.

How do your materials help make better antireflection coatings?

Antireflection coatings have been around for a long time now. The standard coating is a quarter-wavelength-thick layer, and a feature of most of these is that they have zero reflectivity only at a single wavelength. Although this is good

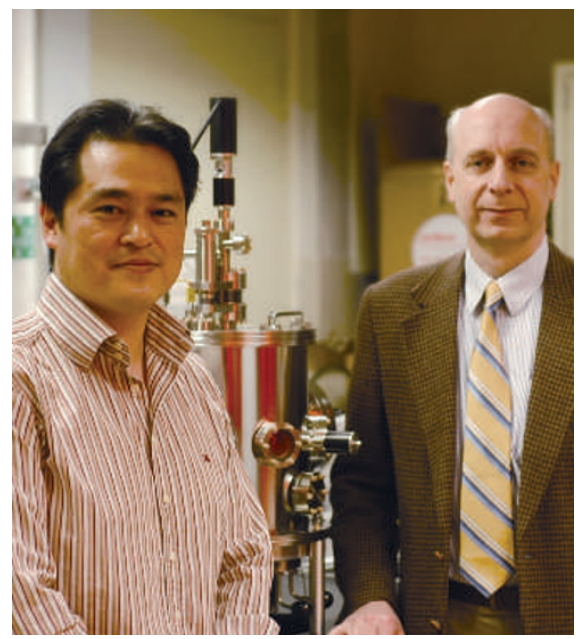

the material to achieve a similar effect. Here an inverted- $\mathrm{V}$ or zigzag shape is etched into the surface, so-called motheye structures - essentially, the aim is to leave very little material at the top surface and leave more and more material the deeper one goes. This approach may not be as controlled as our oblique-angledeposition method because the deeper you go, the effective refractive index or the porosity or the gaps between the structures will change.

What applications do you envisage for your new coatings?

Solar cells could be one possible application. Antireflection coatings increase the amount of light that goes to the active region by a few per cent, which can have a significant impact on performance, especially in large-scale deployments. As for another example, the output efficiency of LEDs is limited by Fresnel reflection between the high-index semiconductor and surrounding air; eliminating this could improve device efficiency. Another application could be in superluminescent diodes - preventing lasing by removing the reflectivity at one facet. On a more fundamental level, the broadband characteristics of our coatings mean they could serve as an experimentally realizable blackbody - a perfect absorber and emitter of radiation. As well as antireflection coatings, these materials can also be used for the opposite purpose: making high-reflectance mirrors, distributed Bragg reflectors made from a single material where the higher index contrast means fewer pairs are necessary. In general, I would say, whenever we have a situation in which we want to get light into or out of a material, these graded-index antireflection coatings would be beneficial. in more reflection than many smaller steps. Our oblique-angle evaporation technique allows us to achieve a very low reflection by depositing five layers of decreasing refractive index, with only a small refractive-index step between each. We are aware of people etching deep into
David Gevaux is an associate editor of Nature Photonics and based in London.

Schubert and his co-workers have a letter on low-refractive-index films on 176 of this issue. 